\title{
Crossmodal congruency effect scores decrease with repeat test exposure
}

\author{
Daniel Blustein ${ }^{\text {Corresp., Equal first author, } 1 \text {, Satinder Gill }{ }^{\text {Equal first author, }{ }^{2} \text {, Adam Wilson }}{ }^{3} \text {, Jon Sensinger }}{ }^{2,3}$ \\ ${ }^{1}$ Department of Psychology and Neuroscience Program, Rhodes College, Memphis, Tennessee, United States \\ 2 Institute of Biomedical Engineering, University of New Brunswick, Fredericton, New Brunswick, Canada \\ 3 Department of Electrical and Computer Engineering, University of New Brunswick, Fredericton, New Brunswick, Canada \\ Corresponding Author: Daniel Blustein \\ Email address: blusteinNeuro@gmail.com
}

The incorporation of feedback into a person's body schema is well established. The crossmodal congruency task (CCT) is used to objectively quantify incorporation without being susceptible to experimenter biases. This visual-tactile interference task is used to calculate the crossmodal congruency effect (CCE) score as a difference in response time between incongruent and congruent trials. Here we show that this metric is susceptible to a learning effect that causes attenuation of the CCE score due to repeated task exposure sessions. We demonstrate that this learning effect is persistent, even after a 6 month hiatus in testing. Two mitigation strategies are proposed: 1 . Only use CCE scores that are taken after learning has stabilized, or 2 . Use a modified CCT protocol that decreases the task exposure time. We show that the modified and shortened CCT protocol, which may be required to meet time or logistical constraints in laboratory or clinical settings, reduced the impact of the learning effect on CCT results. Importantly, the CCE scores from the modified protocol were not significantly more variable than results obtained with the original protocol. This study highlights the importance of considering exposure time to the CCT when designing experiments and suggests two mitigation strategies to improve the utility of this psychophysical assessment. 


\title{
Crossmodal congruency effect scores decrease with repeat test exposure
}

\author{
Daniel Blustein $^{1^{*}}$, Satinder Gill2*, Adam Wilson ${ }^{3}$, Jon Sensinger ${ }^{2,3}$ \\ ${ }^{1}$ Department of Psychology and Neuroscience Program, Rhodes College, Memphis, TN, USA \\ ${ }^{2}$ Institute of Biomedical Engineering, University of New Brunswick, Fredericton, NB, Canada \\ ${ }^{3}$ Department of Electrical and Computer Engineering, University of New Brunswick, \\ Fredericton, NB, Canada \\ *These authors contributed equally to this work. \\ Corresponding author: \\ Daniel Blustein ${ }^{1}$
}

Email: blusteind@,rhodes.edu

\begin{abstract}
The incorporation of feedback into a person's body schema is well established. The crossmodal congruency task (CCT) is used to objectively quantify incorporation without being susceptible to experimenter biases. This visual-tactile interference task is used to calculate the crossmodal congruency effect (CCE) score as a difference in response time between incongruent and congruent trials. Here we show that this metric is susceptible to a learning effect that causes attenuation of the CCE score due to repeated task exposure sessions. We demonstrate that this learning effect is persistent, even after a 6 month hiatus in testing. Two mitigation strategies are proposed: 1 . Only use CCE scores that are taken after learning has stabilized, or 2. Use a modified CCT protocol that decreases the task exposure time. We show that the modified and shortened CCT protocol, which may be required to meet time or logistical constraints in laboratory or clinical settings, reduced the impact of the learning effect on CCT results. Importantly, the CCE scores from the modified protocol were not significantly more variable than results obtained with the original protocol. This study highlights the importance of considering exposure time to the CCT when designing experiments and suggests two mitigation strategies to improve the utility of this psychophysical assessment.
\end{abstract}




\section{Introduction}

There is an increasing interest concerning how the human brain represents the space

41

42

surrounding its body due to converging findings from several different disciplines. The crossmodal congruency task (CCT) is a visual-tactile interference task that has been used to investigate multisensory representation of space in humans (Spence, Pavani \& Driver, 1998; Spence, Pavani \& Driver, 2004; Spence et al., 2004), including those with brain damage (Spence et al., 2001a). Investigation of crossmodal selective attention has been used to demonstrate the detrimental effects of age on the ability to ignore irrelevant sensory information when attending to relevant sensory information (Poliakoff et al., 2006). Other studies have investigated changes in the representation of peripersonal space that are elicited by the prolonged use of hand-held tools (Maravita et al., 2002; Holmes \& Spence, 2004; Holmes, Calvert \& Spence, 2007). Further studies used this task to extend findings regarding physical and pointing tools to virtual robotic tools using techniques from haptics and virtual reality (Sengül et al., 2012; Sengül et al., 2013). Recently it has been used in conjunction with the rubber hand illusion paradigm to investigate the degree of incorporation of a rubber hand into a person's body schema (i.e. how strongly the rubber hand is experienced as one's own hand) (Zopf, Savage \& Williams, 2010; Zopf, Savage \& Williams, 2013).

Although the CCT assessment has become a well-established psychophysics method used to quantify the degree of feedback incorporation in a variety of contexts, in this study we focus on visuo-tactile integration, motivated by its application in the field of neuroprosthetics (Spence, 2015). In such investigations, a CCT typically consists of a participant holding two foam blocks in either hand with vibrotactile targets and visual distractors embedded in the top and bottom of each foam block. A trial consists of a random and independent presentation of a single vibrotactile 
62 target paired with a single visual distractor from any of the four possible pairs of locations.

63 Participants are instructed to make a speeded response regarding the elevation of the vibrotactile

64 target (i.e. "up", at the index finger verses "down", at the thumb), while simultaneously ignoring

65 visual distractors (Spence et al., 2001a).

66 The CCT is a simple stereotypical behavioral task that provides a robust performance metric

67 of feedback incorporation. Participants are typically slower at discriminating the elevation of

68 vibrotactile targets when visual distractors are presented from an incongruent elevation (i.e. when

69 vibrotactile target and visual distractors are presented from different elevations) as compared to a

70 congruent elevation. The crossmodal congruency effect (CCE) score is calculated as the difference

71 in the reaction time between incongruent and congruent trials and is used as a quantitative

72 performance metric for multisensory representation of space. It has been demonstrated that CCE

73 scores are typically higher when the spatial separation between visual distractor and vibrotactile

74 targets is low (e.g. both locations on same block vs. locations on two different blocks held in

75 different hands) (Maravita, Spence \& Driver, 2003; Spence, Pavani \& Driver, 2004).

76 Despite the widespread use of the crossmodal congruency task in a variety of experimental

77 paradigms, important questions related to task exposure times remain unanswered. For example,

78 in a majority of the studies the experimental protocol consists of multiple blocks of trials with

79 experimental sessions lasting up to approximately 60 minutes. These hour-long time-intensive

80 experimental sessions might result in extended learning of the task and adversely affect a

81 participant's CCE score. There is also no specific mention of participant selection criteria based

82 on previous knowledge of the CCT. To our knowledge, there exist no studies to date that have

83 investigated modulation of CCE score due to repeated task exposures. 
Various neuropsychological and cognitive assessments have shown profound learning

85

effects with repeat exposures, including various reaction time tasks (Collie et al., 2003), cognitive function tasks such as the Paced Auditory Serial Addition Test (Beglinger et al., 2005), and other interference tasks such as the Stroop Color and Word Test (Davidson, Zacks \& Williams, 2003; Beglinger et al., 2005). If a task learning or practice effect exists for the CCT, results from previous studies that overlooked such an effect may be inaccurate. Participants tested with previous CCT experience would be expected to have lower CCE scores than participants naïve to the assessment. Any study that presents more than one CCT exposure to a participant may lead to results affected by a learning effect. One previous study repeatedly ran the CCT with a single subject, Patient J.W. (Spence et al., 2001b). Spence et al. only directly compared performance within counterbalanced sessions in order to reduce the effect of differential motivation or fatigue (2001b). However, there is no discussion of a task learning effect, the results from five different sessions across two days are presented side-by-side (Spence et al., 2001b), and the same patient J.W. appears in another CCT study (Spence et al., 2001a). Additionally, studies utilizing a counterbalanced within-subjects design may average out the learning effect, such as Holmes, Calvert \& Spence (2007) in which each participant completed six blocks of 96 trials each across three different counterbalanced conditions. A learning effect, if present, would conflate the research findings that form the basis of our current understanding of multisensory space representation in humans. In the present study we show evidence that CCE scores decrease with repeated task exposure sessions and that this learning effect is persistent over time. We propose two mitigation strategies, including a shortened CCT protocol that we compare to the established protocol. Best practices for future CCE score use are suggested. 
108 We first sought to characterize any change in CCE scores and performance with repeat exposure

109 to the psychophysics protocol.

110

\section{Materials and Methods}

\section{Participants}

113 Twelve healthy volunteer participants were recruited from the local community (11 male, 1

114 female; age mean $\pm \mathrm{SD}=23.4 \pm 6.6$ years; 11 right hand dominant, 1 reported no particular hand

115 dominance). All participants had normal or corrected to normal vision, no disorder of touch, were

116 able to use both foot pedals, and were naïve to the CCT without previous exposure to or knowledge

117 of the task. Participants were informed about the general purpose of the research and were given

118 the opportunity to ask questions but they were not aware of the specific goals of the research.

119 Participants received no compensation. Experiments were conducted under human ethics approval

120 of the U.S. Department of the Navy's Human Research Protection Program and the University of

121 New Brunswick (Fredericton, NB, Canada) Research Ethics Board (Protocol \#2016-032). Written

122 informed consent was obtained from each participant before conducting experiments and no

123 compensation was provided to participants.

124

\section{Materials and apparatus}

126 The experimental test platform was designed around the National Instruments (NI) myRIO

127 embedded hardware system to achieve millisecond-timing accuracy. The three digital outputs of 128 the myRIO system were used to drive one $3 \mathrm{~mm}$ green LED, termed the fixation LED, and two

$1293 \mathrm{~mm}$ green LEDs named distractor LEDs. Two 308-107 Pico Vibe 8mm vibratory motors from 
130 Precision Microdrives were used as the vibrotactile targets for the thumb and index finger. The

131 analog outputs of the myRIO system were used to drive STMicroelectronics L272 power

132 operational amplifiers that drove the vibratory motors. The amplifiers were necessary to provide

133 sufficient driving current to the vibratory motors. Two OnStage KSP100 keyboard sustain pedals

134 were interfaced to the myRIO system through digital inputs to measure a participant's speeded

135 responses to the vibrotactile targets. NI LabVIEW was used to develop the firmware for the myRIO

136 system to randomly activate the visual distractors and vibrotactile targets at a fixed interval and

137 measure the speeded response time. A desktop NI LabVIEW graphical user interface (GUI) was

138 designed to interact with the myRIO embedded system, allowing the experimenter to set various

139 experimental parameters such as vibratory stimulus amplitude, the number of trials and to specify

140 the filename to record the timing results of speeded responses.

\section{Experiment design}

143 In Experiment 1 we assessed the learning effect over five sessions of the CCT. Each of the

144 twelve participants, naïve to the CCT, completed five consecutive days of CCT testing using the

145 standard 8-block protocol (Spence, Pavani \& Driver, 2004). The number of sessions and subjects

146 was determined with a power analysis using preliminary CCT results from eight pilot subjects (see

147 Appendix). For each subject, testing was scheduled at the same time each day for all five visits.

148 The testing time across participants was variable to accommodate different individual schedules.

150 CCT implementation

151 During CCT testing, participants sat comfortably on a chair in front of a table in a dimly

152 illuminated room. They wore over-ear noise-canceling headphones playing Brownian noise to 
153 mask background noise. Identical vibrotactile stimulation motors were placed on the thumb and

154 index fingertips of the participant's right hand (see the Materials and Apparatus section above for

155 hardware details). Distractor light emitting diodes (LEDs) were placed on the thumb and index

156 finger of the participant's right hand, while a fixation LED was centered between the distractor

157 LEDs using a plastic mounting strip. Figure 1 shows the system setup used for this experiment.

158 At the beginning of the CCT assessment, participants were instructed to make a speeded 159 response to vibrotactile targets that were presented randomly to either the thumb or index fingertip.

160 Participants responded by pressing the left or right foot pedals to indicate stimulation of the thumb

161 or index finger, respectively. Participants were explicitly instructed to respond as fast as possible,

162 while making as few errors as possible. Participants were also informed that visual distractors

163 would be presented simultaneously with the vibrotactile targets but that they were completely

164 irrelevant to the vibrotactile target discrimination task. They were specifically instructed to ignore

165 visual distractors by keeping their eyes open and fixating their vision on the central fixation LED.

166 The fixation LED was presented $1000 \mathrm{~ms}$ before the vibrotactile targets and visual distractors were

167 simultaneously presented for $250 \mathrm{~ms}$.

168 The CCT test session was preceded by a practice session consisting of three blocks of 10

169 trials each. The first block of the practice session consisted of presenting the fixation LED and

170 vibrotactile targets only (i.e. no visual distractors were presented) so that participants could

171 familiarize themselves with the vibrotactile target discrimination. We calculated a generalized

172 reaction time for each participant as the mean of the last 5 trials of the first practice block. The

173 visual distractors were presented along with vibrotactile targets in the second and third blocks of

174 the practice session. This was followed by the experimental session during which 64 trials were

175 presented to each participant during each block of trials. Each trial ended when the participant 
176 responded by pressing one of the foot pedals or no response was made within $1500 \mathrm{~ms}$ of target

177 onset.

178 All CCT sessions consisted of eight blocks of 64 trials each. Each block lasted for

179 approximately four minutes and a break period of two minutes was provided between consecutive 180 blocks.

181

182 Analysis

183 All analyses were conducted using custom scripts in MATLAB software (2017 release, The

184 MathWorks, Inc., Natick, MA, USA). Practice session trials were not analyzed except to calculate

185 generalized reaction time. To calculate the CCE score from the test trials, we first discarded trials

186 with an incorrect response, trials with a premature response (i.e. reaction time less than 200ms),

187 and trials with a delayed response (i.e. reaction time greater than $1500 \mathrm{~ms}$ ) as these conditions most

188 likely occurred due to lapses in attention (Spence, Pavani \& Driver, 2004; Sengül et al., 2012).

189 The remaining trials in each block were used to calculate the mean congruent and mean

190 incongruent reaction times. The CCE score for each block was calculated by taking the difference

191 between mean incongruent and congruent reaction times. The mean of the block CCE scores was

192 used to calculate the CCE score for each participant for a particular exposure session. Selection

193 error rates were used as a separate metric of analysis. We evaluated attenuation across sessions

194 using a one-way repeated-measures ANOVA. All data are available on Dryad at 195 doi:10.5061/dryad.150v8g3. 
199 Results - Experiment 1: CCE score decreases over repeated exposures

200 We measured the CCE score of 12 subjects across five sessions using the conventional CCT

201 method of 8-blocks. We observed a significant effect of exposure number on CCE score

202 determined with a repeated-measures ANOVA with Greenhouse-Geisser correction (Fig. 2)

$203(\mathrm{~F}(2.00,21.95)=6.93, \mathrm{p}=.005$, partial-eta=.39). Tests of within-subjects polynomial contrasts over

204 session number indicated a significant linear trend $(F(1,11)=8.63, p=.013)$, a significant $4^{\text {th }}$ order

205 trend $(F(1,11)=5.14, p=.044)$, and a quadratic trend $(F(1,11)=4.83, p=.05)$. Post-hoc comparisons

206 with Bonferroni adjustments showed no significant pair-wise differences. The statistically-

207 supported trends match the observed initial decrease in CCE score and subsequent stabilization,

208 indicating a task learning effect (Fig. 2).

209 To verify that the change in CCE score was due to a learning effect and not attributed to

210 other interacting factors such as variability in motivation or baseline reactivity, we analyzed the

211 CCT selection error rates, generalized reaction times measured from practice trials, and overall

212 reaction times for congruent and incongruent stimuli. We found no significant effect of exposure

213 number on CCT correct trial rate (Fig. 3A), as indicated by a repeated-measures ANOVA with

214 Greenhouse-Geisser correction $(\mathrm{F}(1.15,12.66)=0.53, \mathrm{p}=.51)$. The correct trial rates on

215 congruent and incongruent trials independently did not show consistent trends (Fig. S1).

216 Repeated-measures ANOVA with Greenhouse-Geisser correction indicated a significant effect

217 of exposure on both incongruent reaction time $(F(2.16,23.78)=13.57, \mathrm{p}<.01)$ and congruent

218 reaction time $(\mathrm{F}(1.46,16.07)=8.89, \mathrm{p}<.01)(\mathrm{Fig} .3 \mathrm{~B})$. Although mean generalized reaction time

219 during the practice trials decreased across each session, this trend was not statistically significant

220 as indicated by a repeated-measures ANOVA with Greenhouse-Geisser correction

$221(\mathrm{~F}(2.62,28.81)=2.59, \mathrm{p}=.079)($ Fig. S2). To summarize, the only metrics presenting similar 
222 exposure-dependent decreases as the CCE scores (Fig. 2) were the congruent and incongruent

223 reaction times (Fig. 3B).

224

225

$\underline{\text { Experiment 2 }}$

After observing a statistically significant CCT learning effect in both incongruent and congruent reaction times but not in error rates and generalized reaction times in Experiment 1, we wanted to see if the learning effect persisted over longer time periods. In Experiment 2, we assessed the persistence of the CCT learning effect using a within-subjects design comparing the responses of subjects tested in Experiment 1 with their CCE results following the same protocol 231 about six months later.

\section{Materials and Methods}

All twelve participants from Experiment 1 were asked to return for follow-up testing on a volunteer basis after six months with no CCT exposure. Eight return participants completed Experiment 2 ( 8 male; age mean $\pm \mathrm{SD}=22.3 \pm 7.0$ years; 7 right hand dominant, 1 reported no particular hand dominance). All experimental parameters were the same as in Experiment 1 including the CCT testing apparatus, practice sessions, and test protocol including numbers of an intraclass correlation coefficient analysis [two-way mixed model with single measurements]

241 and calculated the standard error of measurement $[\mathrm{SEM}=\mathrm{SD} \sqrt{ }(1-\mathrm{ICC})]$ to assess the test-retest

242 reliability between the 6 month follow-up visit and the Day 5 visit of Experiment 1 (Weir, 2005). 


\section{Results - Experiment 2: CCT learning effect persists over time}

245 Re-visit CCE scores were similar, and even slightly lower, than the CCE scores measured

246 on Session 5 of Experiment 1, at least 6 months prior [mean time between sessions \pm SD $=201.6$

$247 \pm 9.1$ days; range $=191-209$ days] (Fig. 4). Similarity between Session 5 and the re-visit

248 session was indicated by a high intraclass correlation coefficient $(\operatorname{ICC}(3,1)=.71)$ and a low

249 standard error of measurement $(\mathrm{SEM}=21.6 \mathrm{~ms})$ compared to baseline reliability data calculated

250 by comparing Session 1 and re-visit results $(\operatorname{ICC}(3,1)=.46 ; \mathrm{SEM}=45.7 \mathrm{~ms})$.

\section{$\underline{\text { Experiment } 3}$}

253 The persistence of the learning effect observed in Experiment 2 suggests that CCE scores should 254 only be considered after task learning has stabilized. However, in certain research or clinical 255 settings with subject access or time constraints, extended testing may be impractical. In

256 Experiment 3, we sought to explore mitigation strategies to diminish the impact of the CCT

257 learning effect on research results. Specifically, we tested a modified CCT protocol designed to 258 reduce task exposure to determine if the shortened testing could: 1. Produce valid CCT results; 259 and 2. Mitigate the learning effect.

\section{$\underline{\text { Materials and Methods }}$}

We recruited eighteen volunteer subjects for Experiment 3, all of whom were naïve to the

263 CCT (and had not participated in Experiments 1 and 2). Half of the subjects $(n=9)$ were randomly

264 assigned to test with a shortened CCT protocol ( 3 female; aged $24-57$ years, mean $\pm \mathrm{SD}=33.8$ $265 \pm 10.4$ years; 8 right hand dominant, 1 unspecified hand dominance) and the other nine subjects 
266 tested under the standard protocol used in Experiment 1 and 2 (3 female; aged 20 - 32 years, mean

$267 \pm \mathrm{SD}=26.7 \pm 4.6$ years; 8 right hand dominant, 1 left hand dominant).

268 The testing apparatus was identical to Experiments 1 and 2. In the standard protocol, as

269 described above in the CCT Implementation section, participants completed eight separate testing

270 blocks within each CCT session. In the shortened protocol, we reduced each CCT session to four

271 blocks, with each block still comprised of 64 trials. All other specifications of the CCT

272 implementation were kept consistent with Experiments 1 and 2. We expected fewer CCT trials (in

273 the four block protocol) to result in a reduced learning effect, and we sought to determine if CCE

274 scores would maintain their consistency with the shortened protocol.

275 Participants in Experiment 3 completed two sessions of the CCT, one on each day for two 276 consecutive days. Each participant tested with the same protocol on each day (standard or 277 shortened) and testing sessions were scheduled for each individual at the same time both days. 278 CCE scores were calculated the same way as in Experiments 1 and 2. In Experiment 3 we ran 279 paired t-tests to compare results between the standard and shortened protocol.

281 Results - Experiment 3: Modified CCT protocol reduces learning effect

The shortened protocol reduced the duration of task exposure in each session from eight

blocks to four blocks. In the 4-block protocol, mean CCE score dropped from $117.5 \mathrm{~ms}$ in the first 284 session to $110.0 \mathrm{~ms}$ in the second session (Fig. 5). This was a smaller reduction than observed with the standard 8-block protocol: $114.6 \mathrm{~ms}$ in the first session dropped to $99.9 \mathrm{~ms}$ in the second session (Fig. 5). For the 8-block protocol, mean CCE score dropped by $14.7 \mathrm{~ms}$ from the first exposure to the second, compared to a 7.6ms drop for the 4-block protocol. Variability in the second session was only slightly higher for the 4-block protocol $(\mathrm{SD}=43.8 \mathrm{~ms})$ compared to the 8-block protocol 
$289(\mathrm{SD}=39.0 \mathrm{~ms})$. The variability difference was more pronounced when analyzing the first session 290 results [4-block SD $=64.9 \mathrm{~ms} ; 8$-block $\mathrm{SD}=47.5 \mathrm{~ms}$ ]. None of these differences were statistically

291 significant (paired t-tests, $\mathrm{p}>.05$ ), however.

292

293

294

295

296

297

298

299

300

301

302

303

304

305

306

307

308

309

310

\section{Discussion}

The CCT is a well-established quantitative measure of feedback incorporation that has important implications for objective human sensorimotor assessments (Spence, Pavani \& Driver, 1998; Spence, Pavani \& Driver, 2004; Spence et al., 2004; Spence, 2015). The resulting scores have been used to quantify the rubber hand illusion (Zopf, Savage \& Williams, 2010) and show promise for assessing neuroprosthetic devices (Spence, 2015). Many applications of the CCT require repeated testing - for example, testing the same subject under different sensory feedback conditions to see which one enables the highest degree of feedback incorporation. Oftentimes in research or clinical settings time allotted for sensorimotor assessment can be limited. This study sought to characterize CCT performance through repeat testing to inform its future implementation.

In Experiment 1, we found that with repeat testing, CCE scores decreased over the initial exposure sessions. Different motivation levels or strategies fail to explain the CCE score trend as $\mathrm{CCT}$ error rates did not show a trend matching the decreasing scores. The decreasing trend in generalized reaction times, although not statistically significant, would likely affect both incongruent and congruent reaction times similarly. Thus, we consider the decrease in CCE score to be evidence of a task learning effect. Interestingly, we observed no significant pair-wise differences between CCE scores on different days, even though our statistical tests showed overall 
311 significant effects. This was likely due to the large number of pairwise comparisons and the

312 conservative Bonferroni correction we applied.

313 Although other psychophysical paradigms similarly show significant practice effects

314 (Collie et al., 2003; Davidson, Zacks \& Williams, 2003; Beglinger et al., 2005), for the case of the

315 CCT, scores tend to increase with increased training durations (Blustein, Wilson \& Sensinger

316 2018). The observation of a learning effect in light of these counteracting processes suggests that

317 either an increase in CCE score does not occur with repeat assessment (rather than generalized

318 training), or the learning effect has a much greater impact in the resulting CCE score.

319 In Experiment 2, we demonstrated that the learning effect is persistent as CCE scores after

320 six months remained near the stabilized scores observed at the end of five days of consecutive

321 testing. The persistence of the learning effect provides an opportunity for researchers to only use

322 CCE scores collected after learning has stabilized.

323 In certain circumstances, additional testing to arrive at stable CCE scores may not be

324 feasible. In Experiment 3, we showed that a shortened CCT protocol with four testing blocks

325 instead of eight reduced the impact of the learning effect across two testing sessions. Experiment

3263 was underpowered statistically and thus these findings can only be considered as preliminary

327 trends. In certain circumstances where repeat testing is required but time constraints exclude the

328 possibility of testing until learning asymptotes, the reduced exposure protocol may be necessary.

329 Importantly, CCE score variability did not substantially increase with the 4-block protocol,

330 although the statistical caveats apply here as well.

331 Reducing the number of blocks per session has the added benefit of reducing fatigue,

332 improving the ability of subjects to concentrate over the duration of the study, and shortening the

333 length of the session to approximately 30 minutes (which in turn makes it viable to test a greater 
334 number of subjects or for use in a clinical setting). Using a modified version of the CCT with only

335 four blocks of 64 trials has many potential benefits, with the only drawback identified being a

336 slight increase in the variability of results.

337 Through this characterization of the learning effect associated with the CCT, we have 338 provided support for two distinct mitigation strategies. If extended testing is feasible, researchers 339 can provide enough practice so that CCE scores are only measured and compared after task 340 learning has stabilized. This would be of particular use when tracking an individual's progress 341 over time with repeat testing. In situations where reduced testing is required, researchers can use

342 the 4-block CCT protocol to reduce overall task exposure and subsequently reduce the impact of

343 the learning effect on the results. It is important that these strategies not be mixed; CCE scores

344 should only be compared to other scores that were collected in the same manner. Randomizing

345 condition order to provide counterbalanced groups can also serve to reduce the learning effect's

346 impact on results and can be useful in observing group differences (Holmes, Calvert \& Spence,

347 2007). However, this counterbalancing approach will effectively average out the learning effect,

348 which would lead to reduced magnitudes of any observed differences between conditions.

349 It is not obvious what is being 'learned' through repeated task exposure. We suspect that

350 subjects strengthen an internal model of the foot pedal location tied to the vibration stimulus,

351 resulting in faster response times. Anecdotal support for this idea comes from one subject's

352 comment: "When the feedback is in the opposite location to the light, I sometimes find myself

353 thinking about which pedal to press only to realize I've already pressed the correct pedal".

354 Somatosensory reorganization of an individual's body schema through training may also be

355 implicated (Cardinali et al., 2009). 
357 than scores reported by other researchers (Spence, Pavani \& Driver, 2004; Zopf, Savage \&

358 Williams, 2010). One potential explanation for this observation is that other studies may have

359 different baseline CCE scores due to the experimental inquiry, such as studies using spatial

360 misalignment (Spence, Pavani \& Driver, 2004) or artificial hands (Zopf, Savage \& Williams, 2010;

361 Marini et al., 2014). We would expect a lower degree of feedback incorporation, and thus a lower

362 CCE score (Maravita et al., 2002), under these conditions compared to the ideal conditions we

363 used with both vibratory feedback and visual distractors aligned in place on one of the subject's

364 hands. Additionally, different experimental set-ups could account for differences in CCE scores.

365 Others have recorded user inputs differently, for example with a rocking heel-toe foot pedal setup

366 (Spence, Pavani \& Driver, 2004) or with finger presses (Zopf, Savage \& Williams, 2010). Other

367 variable techniques include the exclusion of trials with extensive eye movement [over 9\% omitted

368 in one study (Spence, Pavani \& Driver, 2004)], or the use of no-go trials as a control (Zopf, Savage

$369 \&$ Williams, 2010). Furthermore, differences in the duration and parameters of practice or

370 familiarization trials before testing could lead to differences in the resulting CCE scores. In a

371 hypothetical experimental setup with all other factors held constant, this study would suggest that

372 a longer practice phase could result in a more pronounced learning effect before testing began,

373 resulting in a lower CCE score. It might also be helpful to explore more granular trial-to-trial

374 variation in the CCT results using mixed effects modeling.

375 Another possible explanation for the observed variability in CCE scores across studies is

376 the different methods used to apply feedback. Different feedback modalities result in different

377 CCE scores (Mayer et al., 2009; Frings \& Spence, 2010). Even when comparing studies using

378 vibration feedback, the method of application can vary and may result in CCE score differences. 
379 We used small vibratory motors $(0.8 \mathrm{~cm}$ diameter vibrating surface) but others have used larger

380 bone conduction vibrators [1.6cm x 2.4cm vibrating surface, (Spence, Pavani \& Driver, 2004)] or

381 small speakers [0.9cm diameter vibrating surface, (Zopf, Savage \& Williams, 2010)], all could

382 result in different degrees of feedback incorporation. To focus on the learning effect, we kept all

383 testing parameters in this study constant. Although we expect these results to be similar across

384 other CCT implementations, the magnitude of the learning effect with different CCT parameters 385 is unknown.

386 Differences in subject characteristics could also explain differences in CCE scores. All of

387 the subjects in this study were initially naïve to the CCT, but it is unclear if that was an inclusion

388 criteria in other studies. Even comparing the first session results from this study across the two

389 cohorts of subjects tested with 8 CCT test blocks we see differences in mean CCE score. In

390 Experiment 1, a mean CCE score of $140 \mathrm{~ms}$ was observed (Fig. 2) compared to $115 \mathrm{~ms}$ in

391 Experiment 3 (Fig. 5). More careful monitoring of additional potentially confounding variables

392 may be helpful. To summarize, there are lots of factors that could affect CCE score and it is

393 impossible to determine if results reported elsewhere are capturing pre-, mid- or post-learning

394 effect scores.

395 To our knowledge, this is the first study that has demonstrated the modulation of CCE

396 score due to task overexposure. We recognize some limitations with this study that could warrant

397 additional studies. Although we collected a generalized reaction time from practice trials, more

398 sophisticated tracking of mental and physical fatigue would be helpful to better inform our

399 understanding of the observed learning effect. Although CCT testing for individual participants

400 was conducted at the same time of the day for each exposure, no strict guidelines were used to

401 ensure that participants were in similar mental or physical states before each test session. 
402 Nevertheless, the learning effect we have described has important implications for future use of

403 the CCT. Going forward, the learning effect must be considered when measuring CCE scores by 404 only using scores from after learning has stabilized, or by using a modified protocol that reduces 405 exposure to the task.

406

407

\section{Conclusion}

408 This study identified a learning effect with an established psychophysics metric: repeat exposure

409 to the crossmodal congruency effect task resulted in reduced scores. The effect persisted during

410 follow-up testing after a six-month hiatus. This learning effect must be considered when using

411 the CCT. For limited testing, we have presented a modified protocol with reduced trial blocks

412 that can be used to limit task exposure. For ongoing testing where multiple tests are necessary,

413 we suggest researchers allow this learning effect stabilize before relying on CCE scores for

414 comparisons. Consideration of the learning effect is important to properly contextualize CCT

415 results.

416

417

\section{Acknowledgments}

418 We thank Matthew Sampson for assistance in data collection and Noah Mesa for assistance in 419 data analysis.

\section{References}

Beglinger, L.J., Gaydos, B., Tangphao-Daniels, O., Duff, K., Kareken, D.A., Crawford, Fastenau, P.S., \& Siemers, E.R. (2005). Practice effects and the use of alternate forms in 
424

425

426

427

428

serial neuropsychological testing, Archives of Clinical Neuropsychology, 20(4), 517-529. doi:10.1016/j.acn.2004.12.003

Blustein, D., Wilson, A., \& Sensinger, J. (2018). Assessing the quality of supplementary sensory feedback using the crossmodal congruency task. Scientific Reports, 8, 6203. doi:10.1038/s41598-018-24560-3

Cardinali, L., Frassinetti, F., Brozzoli, C., Urquizar, C., Roy, A.C., \& Farnè, A. (2009). Tool-use induces morphological updating of the body schema. Current Biology, 19(12), R478-9. doi:10.1016/j.cub.2009.05.009

Collie, A., Maruff, P., Darby, D., \& McStephen, M. (2003). The effects of practice on the cognitive test performance of neurologically normal individuals assessed at brief testretest intervals. Journal of the International Neuropsychological Society, 9(3), 419-428. doi:10.1017/S1355617703930074

Davidson, D.J., Zacks, R.T., \& Williams, C.C. (2003). Stroop interference, practice, and aging. Neuropsychology, development, and cognition. Section B: Aging, neuropsychology and cognition, 10(2), 85-98. doi:10.1076/anec.10.2.85.14463

Frings, C., \& Spence, C. (2010). Crossmodal congruency effects based on stimulus identity. Brain Research, 1354, 113-22. doi:10.1016/j.brainres.2010.07.058

Holmes, N.P., Calvert, G.A., \& Spence, C. (2007). Tool use changes multisensory interactions in seconds: evidence from the crossmodal congruency task. Experimental Brain Research, 183(4), 465-76. doi:10.1007/s00221-007-1060-7 
444 Holmes, N.P., \& Spence, C. (2004). The body schema and the multisensory representation(s) of peripersonal space. Cognitive Processing, 5(2), 94-105. doi:10.1007/s10339-004-0013-3

446 Maravita, A., Spence, C., \& Driver, J. (2003). Multisensory integration and the body schema: close to hand and within reach. Current Biology, 13(13), R531-9. doi:10.1016/S0960-

Maravita, A., Spence, C., Kennett, S., \& Driver, J. (2002). Tool-use changes multimodal spatial interactions between vision and touch in normal humans. Cognition, 83(2), B25-34. doi:10.1016/S0010-0277(02)00003-3

452 453 9822(03)00449-4

Marini, F., Tagliabue, C.F., Sposito, A.V., Hernandez-Arieta, A., Brugger, P., Estévez, N., Maravita, A. (2014). Crossmodal representation of a functional robotic hand arises after extensive training in healthy participants. Neuropsychologia, 53, 178-86. doi:10.1016/j.neuropsychologia.2013.11.017

Mayer, A.R., Franco, A.R., Canive, J., \& Harrington, D.L.. (2009). The effects of stimulus modality and frequency of stimulus presentation on cross-modal distraction. Cerebral Cortex, 19(5), 993-1007. doi:10.1093/cercor/bhn148

Poliakoff, E., Ashworth, S., Lowe, C., \& Spence, C. (2006). Vision and touch in ageing: crossmodal selective attention and visuotactile spatial interactions. Neuropsychologia, 44(4), 507-17. doi:10.1016/j.neuropsychologia.2005.07.004

Sengül, A., Rognini, G., van Elk, M., Aspell, J.E., Bleuler, H., Blanke, O. (2013). Force feedback facilitates multisensory integration during robotic tool use. Experimental Brain Research, 227(4), 497-507. doi:10.1007/s00221-013-3526-0 
465 Sengül, A., van Elk, M., Rognini, G., Aspell, J.E., Bleuler, H., \& Blanke, O. (2012). Extending 466 the body to virtual tools using a robotic surgical interface: evidence from the crossmodal congruency task. PLoS ONE, 7(12), e49473. doi:10.1371/journal.pone.0049473

Spence, C. (2015). The cognitive neuroscience of incorporation: body image adjustment and neuroprosthetics. In: K. Kansaku, L. Cohen, \& N. Birbaumer (Eds.), Clinical Systems Neuroscience (pp. 151-68). Tokyo: Springer. doi:10.1007/978-4-431-55037-2_9

Spence, C., Kingstone, A., Shore, D.I., \& Gazzaniga, M.S. (2001a). Representation of visuotactile space in the split brain. Psychological Science, 12(1), 90-3. doi:10.1111/1467-9280.00316

Spence, C., Shore, D.I., Gazzaniga, M.S., Soto-Faraco, S. \& Kingstone, A. (2001b). Failure to remap visuotactile space across the midline in the split-brain. Canadian Journal of Experimental Psychology, 55(2), 133-40. doi:10.1037/h0087360

Spence, C., Pavani, F., \& Driver J. (1998). What crossing the hand can reveal about crossmodal links in spatial attention. Abstracts of the Psychonomic Society, 3, 13.

Spence, C., Pavani, F., \& Driver, J. (2004). Spatial constraints on visual-tactile cross-modal distractor congruency effects. Cognitive, Affective, \& Behavioral Neuroscience, 4(2), 148-69. doi:10.3758/CABN.4.2.148

Spence, C., Pavani, F., Maravita, A., \& Holmes, N. (2004). Multisensory contributions to the 3D representation of visuotactile peripersonal space in humans: evidence from the crossmodal congruency task. Journal of Physiology - Paris, 98(1-3), 171-89. doi:10.1016/j.jphysparis.2004.03.008 
486 Weir, J.P. (2005). Quantifying test-retest reliability using the intraclass correlation coefficient 487 and the SEM. Journal of Strength \& Conditioning Research, 19(1), 231-40.

488 doi:10.1519/15184.1

Zopf, R., Savage, G., \& Williams, M.A. (2010). Crossmodal congruency measures of lateral distance effects on the rubber hand illusion. Neuropsychologia, 48(3), 713-25.

491 doi:10.1016/j.neuropsychologia.2009.10.028

492

Zopf, R., Savage, G., \& Williams, M.A. (2013). The crossmodal congruency task as a means to 493 obtain an objective behavioral measure in the rubber hand illusion paradigm. Journal of Visualized Experiments, 77:e50530. doi:10.3791/50530 
Figure 1

Overall system setup.

Subjects make speeded responses with foot pedals to vibratory feedback presented on the index finger or thumb. Subject is asked to focus on the fixation LED while ignoring the distractor LEDs. See text for method details. 


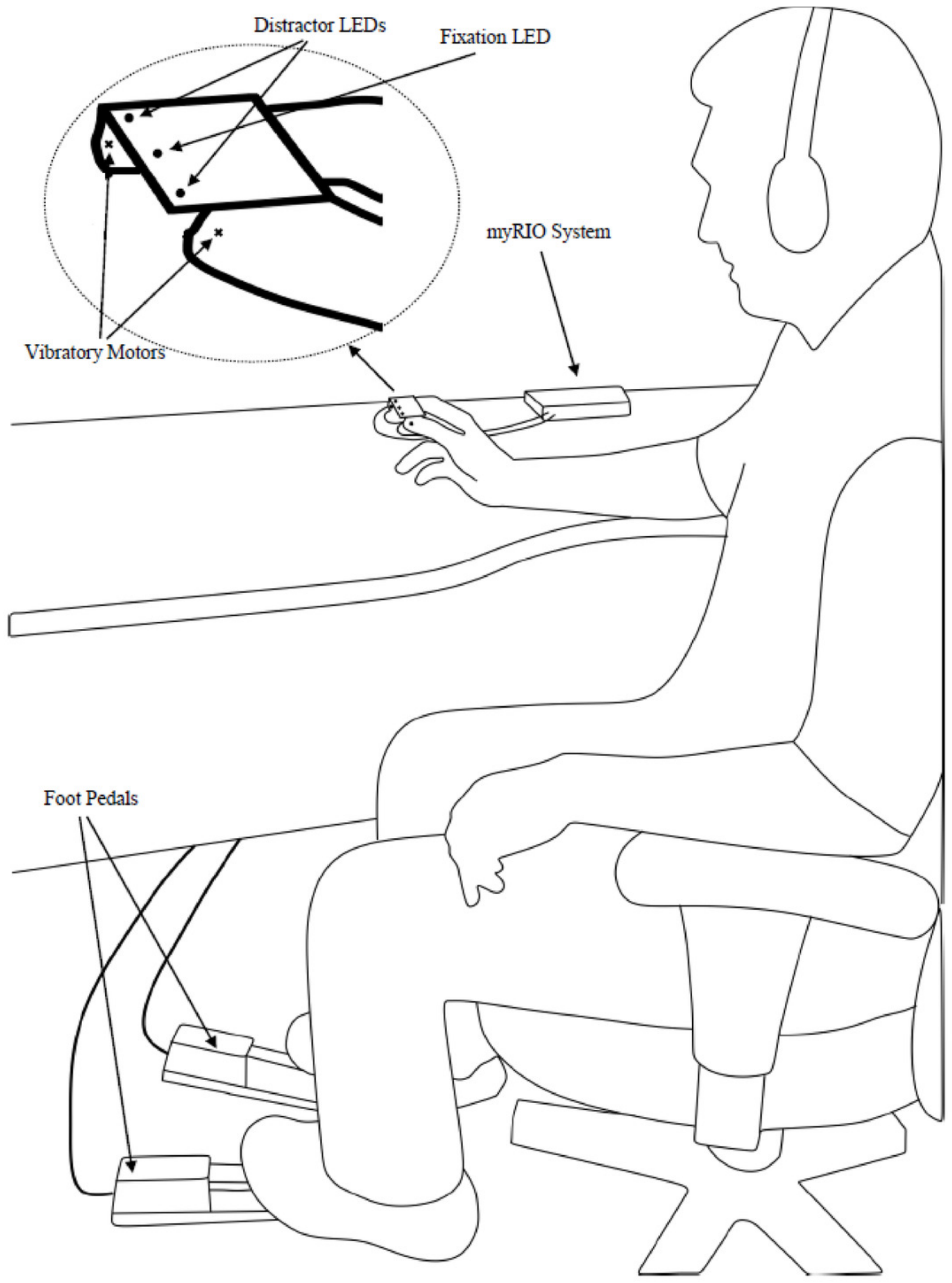

Peer) reviewing PDF | (2018:09:31222:2:1:NEW 17 Apr 2019) 
Figure 2

CCE score decreases with task exposure.

Each data point represents the mean CCE score for 12 subjects. Error bars show standard error. Results from Experiment 1.

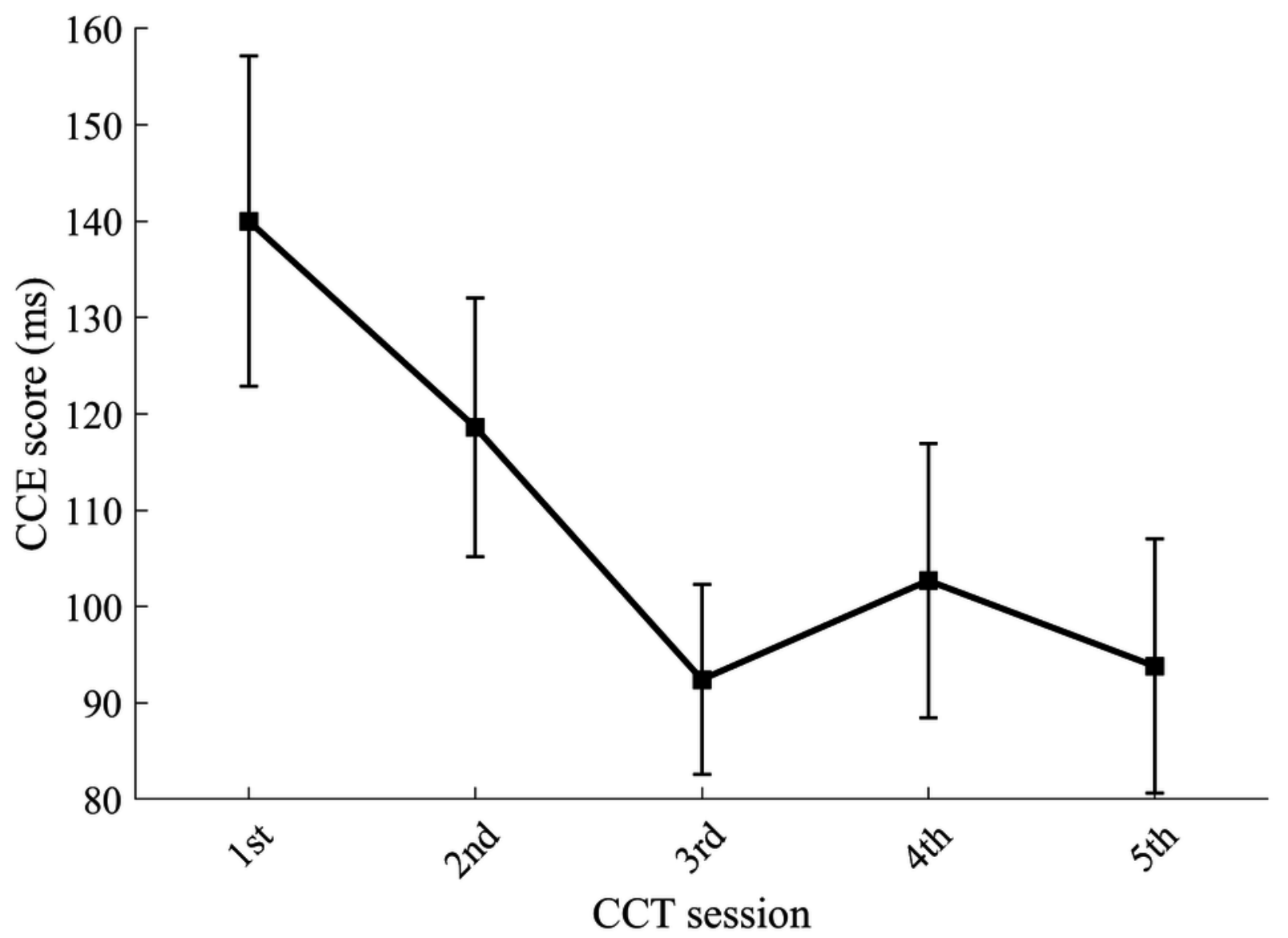


Figure 3

Decreasing CCE score trend matched in isolated reaction times but not in CCT correct trial rate.

A. Plot of proportion correctly selected trials over repeated exposures. An incorrect trial occurred when the wrong pedal was pressed (e.g. vibration stimulus on the thumb resulted in subject pressing the pedal indicating an index finger stimulus). B. Congruent and incongruent reaction times plotted independently. Results from Experiment 1.

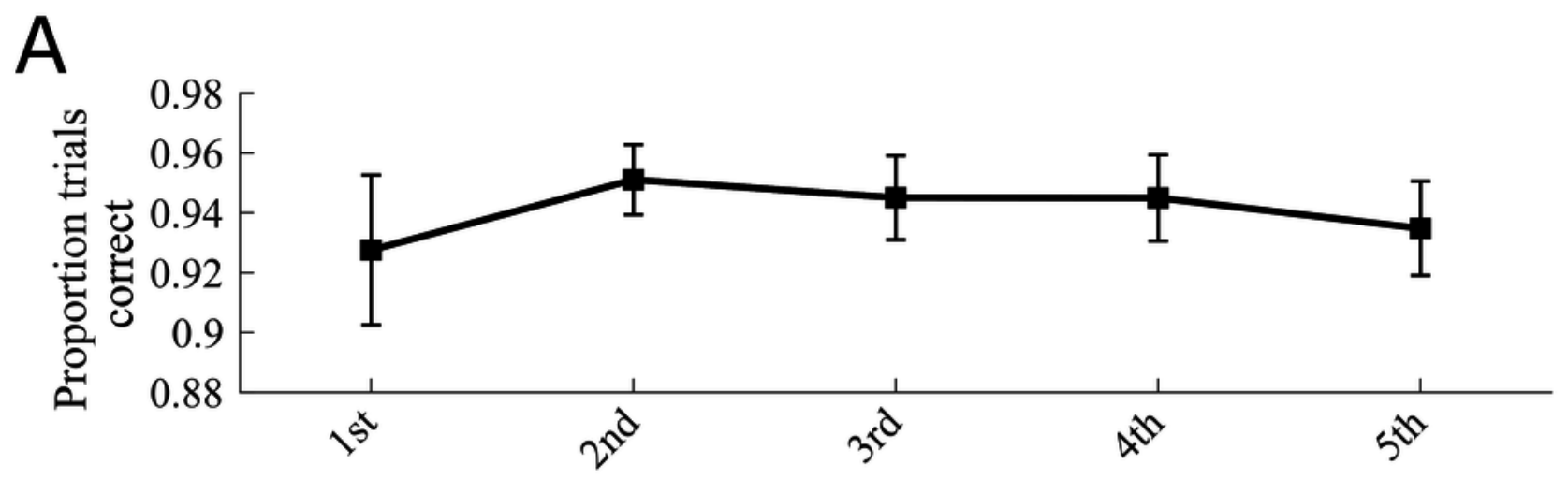

B

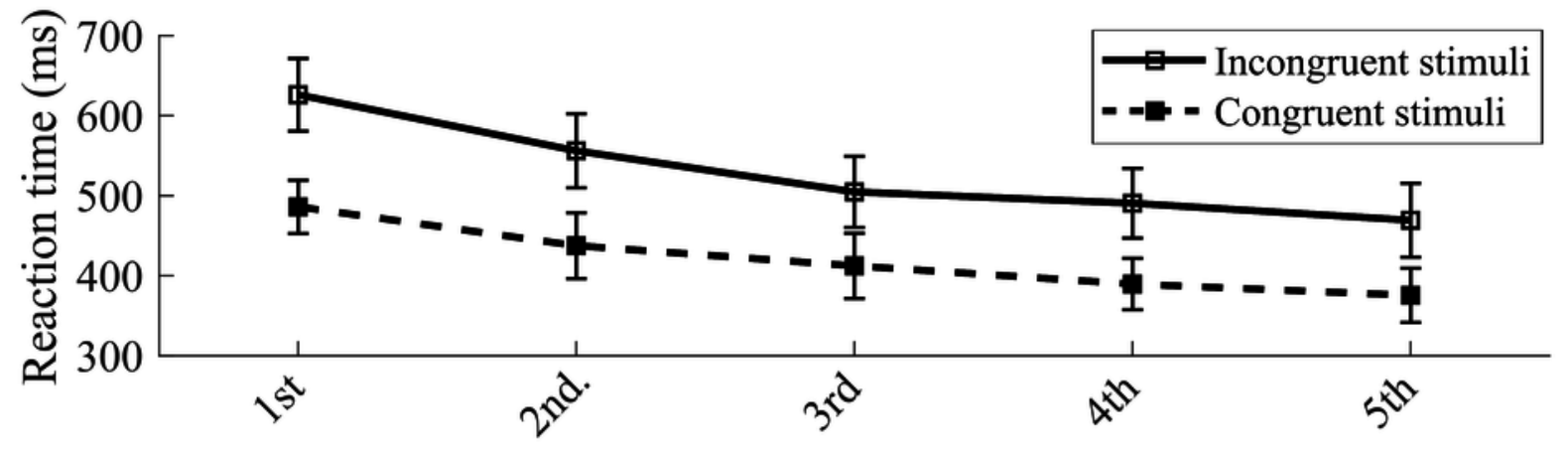

CCT session 
Figure 4

Persistent CCT learning effect after 6-month follow-up testing.

Eight subjects from Experiment 1 returned for a sixth visit about 6 months after their initial visits. Data shown are means and standard error for those eight subjects. Results from Experiment 2.

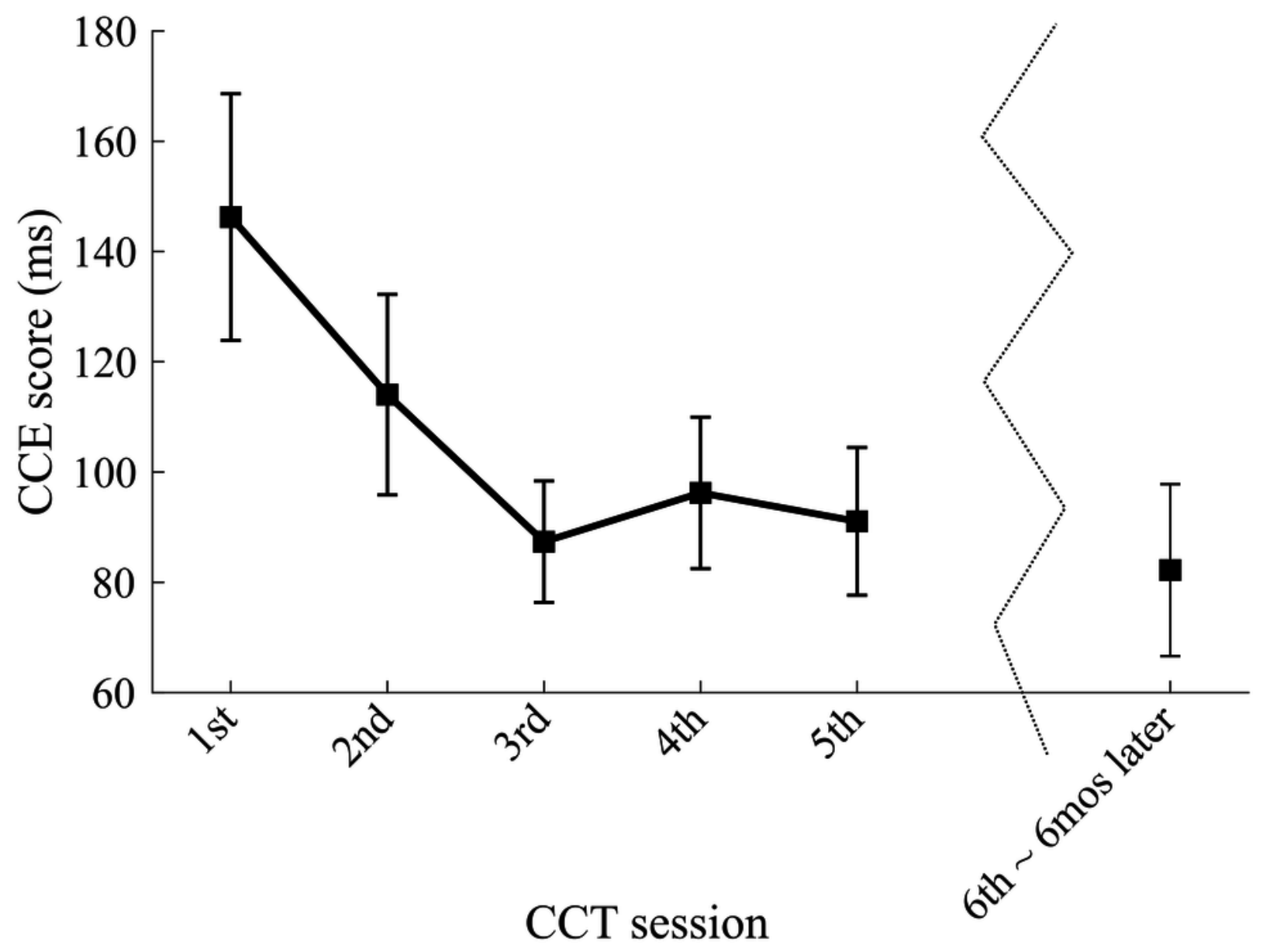




\section{Figure 5}

First exposure vs. second exposure mean CCE scores for standard and shortened protocols.

Means and standard error for 8-block standard $(n=9)$ and 4-block shortened $(n=9)$ protocol participants. Dark-colored bars represent the first exposure session and light-colored bars represent the second exposure session. Results from Experiment 3.

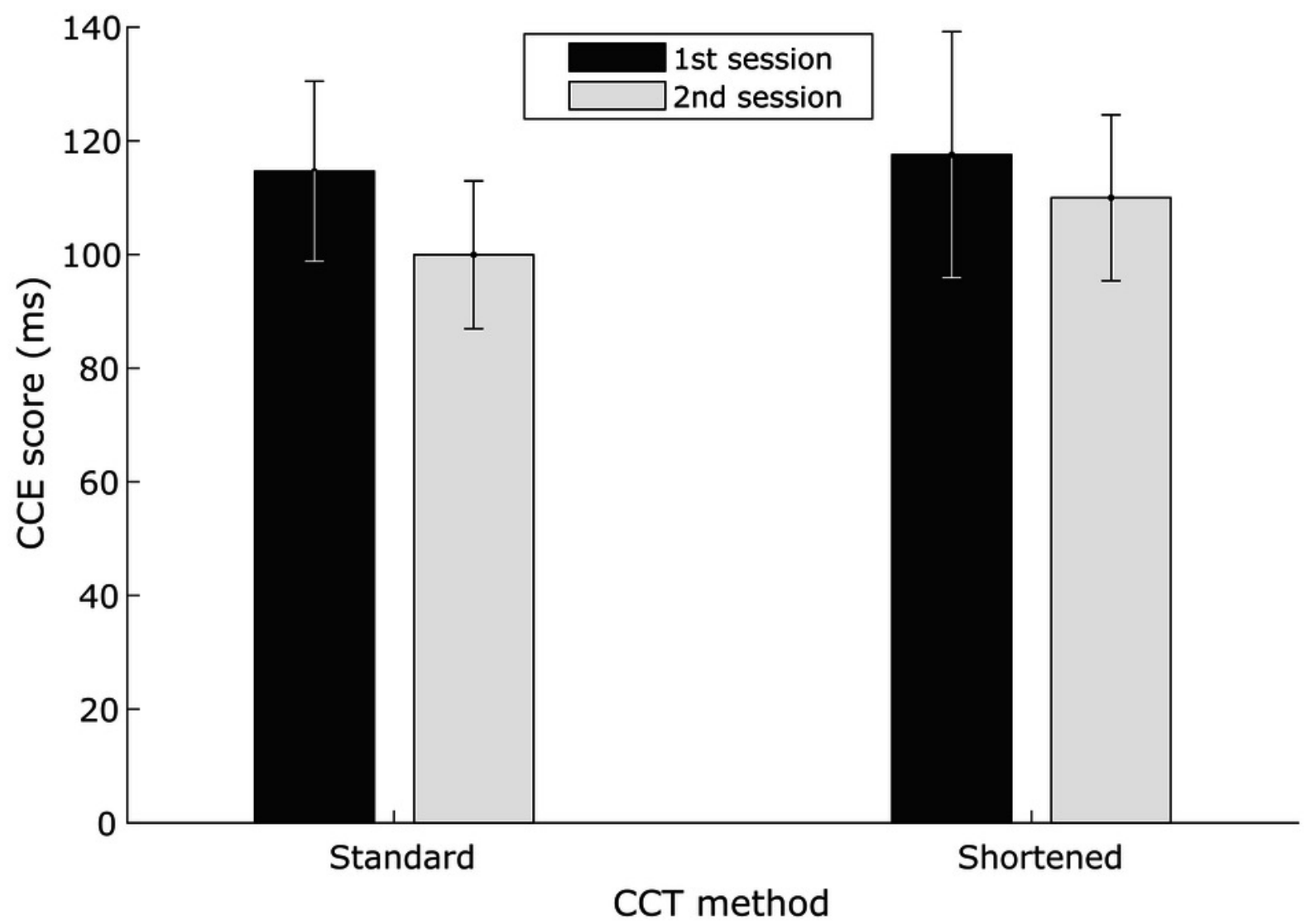

